\title{
Numerical simulations of atomic decay in cavities and material media
}

\author{
V. Bužek, ${ }^{1,2}$ G. Drobný, ${ }^{1}$ Min Gyu Kim,,${ }^{1} *$ M. Havukainen, ${ }^{3}$ and P. L. Knight ${ }^{4}$ \\ ${ }^{1}$ Institute of Physics, Slovak Academy of Sciences, Dúbravská cesta 9, 84228 Bratislava, Slovakia \\ ${ }^{2}$ Faculty of Informatics, Masaryk University, Botanická 68a, 60200 Brno, Czech Republic \\ ${ }^{3}$ Helsinki Institute of Physics, P.O. Box 9, FIN-00014 University of Helsinki, Finland \\ ${ }^{4}$ Optics Section, The Blackett Laboratory, Imperial College, London SW7 2BZ, United Kingdom
}

(Received 30 November 1998; revised manuscript received 1 March 1999)

\begin{abstract}
Starting from first principles we simulate numerically the dynamics of a system of two-level atoms interacting with a multimode electromagnetic field in a one-dimensional cavity. In particular, we investigate the spontaneous emission of an excited two-level atom in the cavity. We show how the decay depends on the mode spectrum and on the position of the atom in the cavity. We study how the spontaneous emission is modified when the initially excited atom is surrounded by a material medium modeled as a system of two-level atoms. We also study the propagation of photon wave packets emitted by the atom within the material media. Our microscopic model provides us with a deeper understanding of the atom-field interaction and offers us a framework for a systematic investigation of the transition from a microscopic to a macroscopic (phenomenological) description of the spontaneous decay in material media (e.g., dielectrics). [S1050-2947(99)04307-3]
\end{abstract}

PACS number(s): 42.50.Ct, 32.80.-t, 32.90.+a

\section{INTRODUCTION}

Quantum electrodynamics (QED) lies at the heart of modern quantum theory. QED is a well established and experimentally confirmed theory $[1,2]$ but even fifty years after its foundation many features of the atom-field interaction remain of interest. In particular, the character of the atom-field interaction can be substantially modified in confined spaces (e.g., within the high- $Q$ cavity of a micromaser) due to the fact that local properties of the electromagnetic (EM) field depend on space boundaries. The radiative properties of atoms and the EM field in confined spaces have been investigated for various cavity QED systems [3-9].

Quantum electrodynamics is a local theory, which means that the dynamics of atoms and electrons depend on local properties of the electromagnetic field with which they interact. But local properties of the electromagnetic field depend also on conditions imposed by the boundaries of the space region in which the field is confined [10]. These conditions are reflected in the quantization of the field. Specifically, we can either quantize the electromagnetic field in a free space or in a "quantization box" of linear dimension $L$. Physically, quantum electrodynamics in a box describes an idealization of effects associated with processes inside high-quality (perfect) cavities. In addition, quantization in a box can be considered as an approximation to the free-space quantization and the two theories must give the same results in the limit $L \rightarrow \infty$.

In their pioneering quantum-mechanical description of the spontaneous decay of a two-level atom in free space, Weisskopf and Wigner [11] started their calculations with the cavity modes quantized in a box, and then at a certain stage of the calculation, the limit of a continuum of modes was taken.

\footnotetext{
*Permanent address: Korea Atomic Energy Research Institute, Laboratory for Quantum Optics, P.O. Box 105, Yusong, Taejeon, South Korea.
}

This approach gives in the first approximation correct results (exponential decay of the excited level of the atom). The interaction of a two level atom with discrete (cavity) modes has been described systematically by Hamilton [12] who solved the emission and scattering problems exactly for a cubic box by diagonalization of the total atom-field Hamiltonian. Later this approach was utilized for a detailed investigation of the spontaneous emission of two-level atoms (see, for instance, papers by Davidson and Kozak [13] and Swain [14]). In all these papers devoted to the spontaneous decay of a two-level atom in a cavity (box), the coupling constant between the atom and the cavity modes was taken to be position independent. This argument is perfectly justified in free space, when translational invariance is valid. On the other hand, when the atom interacts with discrete cavity modes in a confined space the position dependence of the coupling can play a significant rôle. The investigation of this problem is not only of theoretical interest. Recent advances in experimental techniques have allowed one to study fundamental processes in cavity quantum electrodynamics (cavity QED) [3-9] and to verify various effects of the atom-field interaction in confined spaces as predicted by Schelkunoff [15], Purcell [16], Barton [17] and others.

One of the fundamental processes of cavity QED is the spontaneous decay of a two-level atom. It is well known that the spontaneous emission from an atom positioned very close to a cavity mirror can be significantly suppressed. This effect is called inhibition of spontaneous emission [4]. The deviation from the exponential Weisskopf-Wigner decay of an atom in free space [18] has been demonstrated in a number of experiments [9]. Many other interesting questions arise for these QED systems. For example, one could ask what is the influence of cavity mirrors on the dynamics of the atom and the role of the position of the atom on the appearance of Poincaré recurrences (i.e., reexcitations of the atom by the radiation reflected by the cavity mirrors) [19]. While the exponential-like character of the decay during the first stage of the time evolution (see below) is not affected by 
variations in the position of the atom around the center of the cavity (providing the density of the cavity modes is large enough), Poincare recurrences depend very sensitively on the position of the atom inside the cavity. Variation in the position of the atom within a wavelength of the resonant atomic frequency can result in an almost complete suppression of the first Poincare recurrence of the excited level of the atom [19]. This means that the atom effectively does not "feel", wave packets reflected from cavity mirrors for times much longer than the time necessary for the emitted light to "travel" to the mirrors and back to the atom. Another example is that of the atom positioned close to one of the mirrors. In general one may expect to see inhibition of the radiation. Nevertheless, taking into account the position dependence of the field-atom interaction it turns out that for some specific distances from the mirror (e.g., one quarter of the resonant wavelength of the radiation field), the atom decays even faster than in free space [19].

A first insight into the modification of the spontaneous emission of the two-level atom can be obtained with the help of the Fermi golden rule [1]

$$
\Gamma_{a}=\frac{2 \pi}{\hbar^{2}}\left|V_{f i}\right|^{2} \rho\left(\omega_{a}\right)
$$

The spontaneous emission rate $\Gamma_{a}$ is directly proportional to the density of the field modes $\rho\left(\omega_{a}\right)$ at the atomic transition frequency $\omega_{a} ; V_{f i}$ is the matrix element of the corresponding transition. The presence of boundaries (e.g., in the case when the atom is inserted into a high- $Q$ cavity) changes the local density of field modes and thereby the spontaneous emission can be suppressed or enhanced. However, it is not necessary to change the boundary conditions of the EM field in order to modify the spontaneous emission rate. This goal can be achieved when we assume that the excited atom is embedded in a material medium (e.g., a dielectric). In this paper we will model material media as a collection of twolevel atoms initially prepared in their ground state.

The main goal of our investigation is the numerical simulation of the atom-field interaction in confined geometries. Starting from first principles, we simulate the dynamics of a system of atoms in a one-dimensional (1D) cavity. In particular, we investigate the spontaneous emission of an excited two-level atom in the cavity. We show how the decay depends on the mode spectrum and on the position of the atom in the cavity. We study how the spontaneous emission is modified when the initially excited atom is surrounded by a material medium, modeled as a system of two-level atoms. We also study the propagation of photon wave packets emitted by the atom. Our microscopic model provides us with a deeper understanding of the atom-field interaction and offers a framework to study systematically the transition from the microscopic to macroscopic (phenomenological) description of the spontaneous decay in a material medium (e.g., dielectrics). In particular, this will allow us to study dynamics of atoms in photonic band gap structures [20-22].

The paper is organized as follows. We describe the model in Sec. II. In Sec. III we study the position dependence of the decay of a single two-level atom and analyze the spectrum of the emitted radiation. In addition we discuss specific technical questions such as the role of the frequency cutoff. We describe the effect of the inhibition of spontaneous emission in Sec. IV. In Sec. V we analyze the decay of a two-level atom in a material medium modeled as a set of two-level atoms. Finally, in Sec. VI we present the convolutionless master equation describing the dynamics of the initially excited atom within the material medium. We summarize our results in Sec. VII.

\section{THE MODEL}

We consider a simple one-dimensional model of a cavity in which two-level atoms interact with the cavity modes in the dipole and the rotating-wave approximations. To simplify the model, we neglect all mechanical effects of the cavity field on the atom (i.e., the mass of the atom is assumed to be infinite). This 1D model not only reflects the main features of atom-field interaction but also can be mapped onto an isotropic 3D model.

Under the assumption of perfectly reflecting mirrors, the operator of the electric field inside the cavity in the Coulomb gauge can be expressed as $[1,23,24]$

$$
\vec{E}(r)=\sum_{n, \lambda} \mathcal{E}_{n} \vec{e}_{\lambda}\left(\hat{a}_{n, \lambda}+\hat{a}_{n, \lambda}^{\dagger}\right) \sin \left(k_{n} r\right)
$$

where $k_{n}=\omega_{n} / c=n \pi / L$ and $\mathcal{E}_{n}=\sqrt{\hbar \omega_{n} / \epsilon_{0} L}$. The two orthogonal polarization vectors $\vec{e}_{\lambda}(\lambda=1,2)$ lie in the plane perpendicular to the cavity axis; $\hat{a}_{n, \lambda}$ and $\hat{a}_{n, \lambda}^{\dagger}$ are annihilation and creation operators of the $n$th mode.

The Hamiltonian describing the free cavity field can be expressed as

$$
\hat{H}_{F}=\hbar \sum_{\lambda} \sum_{n=1}^{N} \omega_{n} \hat{a}_{n, \lambda}^{\dagger} \hat{a}_{n, \lambda},
$$

where we have omitted the zero-point contribution $\hbar \Sigma_{n} \omega_{n} / 2$. Summation over discrete modes in Eq. (3) is performed only up to $n=N$, which means that in our model we assume a cutoff for the cavity modes (for more discussion see below).

The Hamiltonian describing a set of $M$ noninteracting ("free") two-level atoms with transition frequencies $\omega_{a}^{(j)}$ can be expressed as

$$
\hat{H}_{A}=\frac{\hbar}{2} \sum_{j=1}^{M} \omega_{a}^{(j)} \hat{\sigma}_{z}^{(j)}
$$

where $\hat{\sigma}_{z}^{(j)}=|e\rangle_{j}\langle e|-| g\rangle_{j}\langle g|;| e\rangle_{j}$ and $|g\rangle_{j}$ denote the upper and lower atomic states of the atom at the position $r_{j}$, respectively.

When the radius of the atom is much smaller than the wavelength of the resonant electromagnetic radiation then the atom-field interaction can be described within the electric-dipole approximation, i.e., $\hat{H}_{\mathrm{int}}=-\overrightarrow{\hat{d}} \cdot \overrightarrow{\hat{E}}$. For simplicity we neglect all polarization effects and then the resulting interaction Hamiltonian in the rotating-wave approximation (RWA) reads

$$
\hat{H}_{\mathrm{int}}=-\hbar \sum_{j=1}^{M} \sum_{n=1}^{N} g_{n}^{(j)}\left[\hat{a}_{n} \hat{\sigma}_{+}^{(j)}+\hat{a}_{n}^{\dagger} \hat{\sigma}_{-}^{(j)}\right],
$$


where the Pauli spin-flip operators are $\hat{\sigma}_{+}^{(j)}=|e\rangle_{j}\langle g|$ and $\hat{\sigma}_{-}^{(j)}=|g\rangle_{j}\langle e|$. The position-dependent coupling constants $g_{n}^{(j)}$ are given by the expression

$$
g_{n}^{(j)}=\left(\frac{\omega_{n}}{\hbar \epsilon_{0} L}\right)^{1 / 2} d_{e g}^{(j)} \sin \left(k_{n} r_{j}\right)
$$

where the parameters $d_{e g}^{(j)}$ denote the dipole matrix elements of the atoms. The position dependence of the atom-field coupling constant (6) given by space-mode functions $f_{n}(r)$ $=\sin \left(k_{n} r\right)$ may significantly affect the atomic dynamics.

The total Hamiltonian of the form

$$
\hat{H}_{\text {tot }}=\hat{H}_{F}+\hat{H}_{A}+\hat{H}_{\text {int }}
$$

describes the system of $M$ two-level atoms interacting with $N$ discrete field modes in a 1D cavity. This model can be solved exactly, because the total number of excitations

$$
\hat{R}=\frac{1}{2} \sum_{j=1}^{M}\left(\hat{\sigma}_{z}^{(j)}+1\right)+\sum_{n=1}^{N} \hat{a}_{n}^{\dagger} \hat{a}_{n}
$$

is an integral of motion, i.e., $\left[\hat{R}, \hat{H}_{\mathrm{tot}}\right]=0$.

\section{A. Solution of the model}

In this paper we will consider situations when just one of the atoms is initially excited while the other atoms are in their ground state. The electromagnetic field will be considered to be initially in the vacuum state. In this case we can write the initial state vector in the form

$$
\left|\Psi\left(t_{0}\right)\right\rangle=|e\rangle_{1}|g\rangle_{j}|0\rangle_{\vec{k}},
$$

where $|e\rangle_{1}$ describes the excited state of the initially excited atom, while $|g\rangle_{j}:=|g\rangle_{2} \otimes \cdots \otimes|g\rangle_{M}$ describes the rest of the $M$ atoms which are initially in the ground state. The vector $|0\rangle_{\vec{k}}$ denotes the vacuum of the multimode cavity field. Because the model Hamiltonian $\hat{H}_{\text {tot }}$ (7) is chosen so that the number of excitations given by Eq. (8) in the system is an integral of motion, we can express the state vector of the atom-field system at time $t$ as

$$
\begin{aligned}
|\Psi(t)\rangle= & c_{1}(t)|e\rangle_{1}|g\rangle_{j}|0\rangle_{\vec{k}}+\sum_{j=2}^{M} c_{j}(t)|g\rangle_{1}\left|e_{j}\right\rangle_{j}|0\rangle_{\vec{k}} \\
& +\sum_{k} d_{k}(t)|g\rangle_{1}|g\rangle_{\vec{j}}\left|1_{k}\right\rangle_{\vec{k}},
\end{aligned}
$$

where $\left|e_{j}\right\rangle_{j}$ describes the state vector of a set of $M-1$ atoms out of which the $j$ th atom is excited, while $\left|1_{k}\right\rangle_{\vec{k}}$ describes the state of the cavity field with the $k$ th mode in the Fock state $|1\rangle$ and all other modes in the vacuum state.

In general it is impossible to find a closed analytical solution of the Schrödinger equation for the system under consideration except in a few cases, such as the JaynesCummings model [25] which describes the dynamics of a two-level atom interacting with a single mode cavity field. Therefore we will study the dynamics of our system numerically. We will use two approaches. The first one is based on the straightforward diagonalization of the total Hamiltonian
(7). Here we assume that the Hilbert space of the cavity modes can be truncated (i.e., we effectively apply the frequency cutoff) so that the Hamiltonian under consideration can be represented as a finite matrix. Then we find eigenvalues $E_{j}$ and eigenvectors $\left|\Phi_{j}\right\rangle$ of $\hat{H}_{\text {tot }}$. The state vector $|\Psi(t)\rangle$ can then be written as

$$
|\Psi(t)\rangle=\sum_{j} \exp \left[-i E_{j}\left(t-t_{0}\right)\right]\left|\Phi_{j}\right\rangle\left\langle\Phi_{j} \mid \Psi\left(t_{0}\right)\right\rangle .
$$

This method is conceptually very simple, except it is not very efficient in many cases. Therefore, in some cases we transform the Schrödinger equation for the state vector (10) with the Hamiltonian (7) into a set of coupled linear differential equations for the amplitudes $c_{j}(t)$ and $d_{k}(t)$ (here again the frequency cutoff is applied). The solutions for the amplitudes are then found by standard Runge-Kutta methods [26].

\section{B. Observables}

Using the numerical solutions of the model we analyze the time evolution of the mean values of the following observables.

(i) The occupation of the upper level of the $j$ th atom

$$
\hat{P}_{e}^{(j)}=\frac{\hat{\sigma}_{z}^{(j)}+1}{2}=|e\rangle_{j}\langle e| .
$$

(ii) The amplitude of the electric field

$$
\hat{E}(r)=\sum_{n=1}^{N}\left(\frac{\hbar \omega_{n}}{\epsilon_{0} L}\right)^{1 / 2}\left[\hat{a}_{n}+\hat{a}_{n}^{\dagger}\right] \sin \left(k_{n} r\right) .
$$

(iii) The number of excitations of the $k$ th mode

$$
\hat{S}(k)=\hat{a}_{k}^{\dagger} \hat{a}_{k},
$$

which are used to study the time-dependent spectrum of the electromagnetic radiation in the cavity.

(iv) To analyze the space-time propagation of radiation wave packets, we evaluate mean values of the normallyordered operator for the energy density, which in our case can be written as

$$
\hat{I}(r)=: \epsilon_{0} \hat{E}^{2}(r): .
$$

Here normal ordering (indicated by the colons above) is adopted to eliminate the vacuum-state contribution to the energy density of the emitted radiation.

In what follows we demonstrate the main features of the atom-field interaction in confined geometries. In particular, we will concentrate our attention on two main problems.

(a) Modification of the spontaneous emission of the atom in the cavity due to the position dependence of the atom-field interaction. A partial reexcitation of the atom caused by the back reflected radiation (Poincaré recurrences).

(b) Decay of the two-level atom in a "material media:" modification of the spontaneous emission due to the presence of other atoms, which are initially in their ground states (the decaying atom can be considered as being embedded in a dielectric "crystal" which is formed by other atoms). 


\section{DECAY AND REEXCITATION OF ATOM}

From the Weisskopf-Wigner theory [11] it follows that the initially excited atom coupled to a continuum of the vacuum field modes in free space decays exponentially to its ground state. Representing the usual 1D continuum by a discrete model (5) for a large cavity $(L \rightarrow \infty)$, we find that the population of the excited atomic level $P_{e}$ decays exponentially with a rate $\Gamma_{a}$ given by the Fermi golden rule (1)

$$
P_{e}(t)=\exp \left(-\Gamma_{a} t\right), \quad \Gamma_{a}=\frac{\omega_{a}\left|d_{e g}\right|^{2}}{\epsilon_{0} \hbar c}
$$

In the 1D "free" space model $(L \rightarrow \infty)$ the decay process is accompanied by the emission of two wave packets (representing the one-photon state) propagating to the left and to the right from the atom. In the case of the "left-right" symmetry of atomic-wave functions in 1D (this corresponds to spherical symmetry in the 3D problem) each of the two emitted wave packets carries half of the atomic initial excitation. This process is irreversible, as the energy cannot be reabsorbed by the atom (which is reflected by the exponential decay of the atomic excitation).

In confined geometries we have a different picture. First, the density of the discrete modes is changed due to the boundary conditions. The translational symmetry is lost and the coupling between the atom and the field is positiondependent. In particular, when the atom is positioned at the center of the cavity it is coupled only to the odd modes of the field [for even modes the coupling constant (6) is equal to zero]. Secondly, the two wave packets are reflected back by the cavity mirrors and can be (partially) reabsorbed by the atom. This partial restoration of the initial state of the atom, the so-called Poincaré recurrence, can be viewed as a consequence of constructive quantum interference (see below).

In Fig. 1 we show the time evolution of the probability of the atomic excitation for four different values of the position of the atom around the center of the cavity, namely $\Delta r_{1}$ $\equiv r_{1}-L / 2=0, \pm \lambda_{a} / 16, \pm \lambda_{a} / 8, \pm \lambda_{a} / 4$. From this figure we see that the first "exponential" stage of the decay is (almost) position independent. Providing the atom is "far" from the cavity mirrors, i.e., $\min \left(r_{1}, L-r_{1}\right) \gg c / \Gamma_{a}$, the reflected wave packets do not influence the exponential decay.

We note that the initially excited atom which is positioned in the cavity center (dotted line) interacts only with the odd modes and thus the effective density of modes equals to $L / 2 \pi c$. Using the Fermi golden rule (1) we find that in this case the corresponding decay rate is exactly the same as the free-space decay rate $\Gamma_{a}$ given by Eq. (16). We note that the density of interacting modes is doubled when the atom is shifted from the cavity center. On the other hand the strength of the interaction with the odd modes is weaker [see Eq. (6)]. Specifically, the effective squared interaction constant, equal to the average of the coupling of the atom to the two neighboring (odd and even) cavity modes, is equal to one half of the squared coupling constant between the atom in the center of the cavity and the odd modes. Consequently, even though the atom is shifted from the cavity center the Fermi golden rule (1) with the effective squared interaction constant and doubled density of modes leads to the same decay rate (16). This is nicely illustrated in Fig. 1, from which we see that

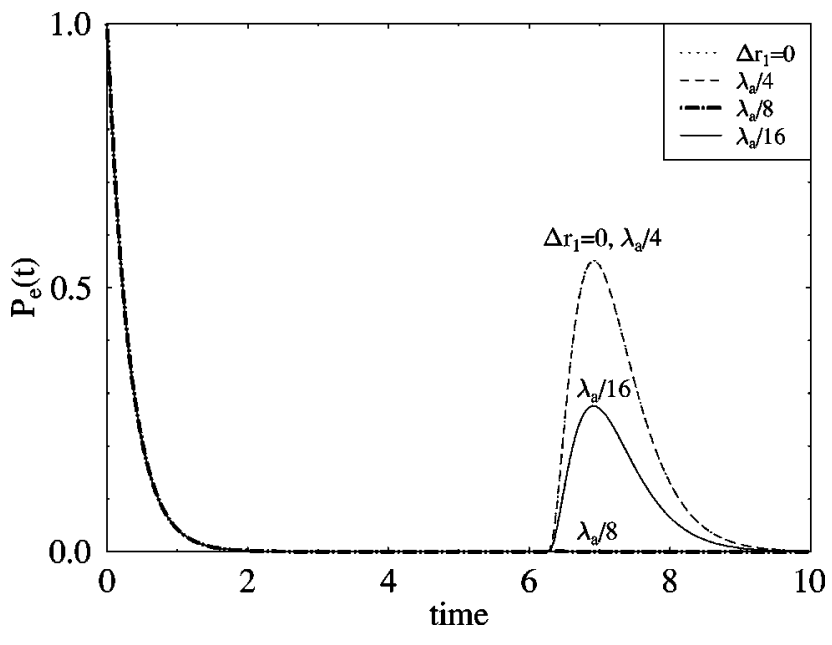

FIG. 1. The time evolution of the population $P_{e}(t)$ of the excited atomic level. The atom is shifted from the cavity center by $\Delta r_{1}=0$ (dotted line), $\Delta r_{1}= \pm \lambda_{a} / 4$ (dashed line), $\Delta r_{1}= \pm \lambda_{a} / 8$ (dashed-dotted line), and $\Delta r_{1}= \pm \lambda_{a} / 16$ (solid line). The atom is initially prepared in its excited state and the multimode cavity field is in the vacuum. The choice of the cavity length (in dimensionless units) $L=2 \pi$, the squared coupling constant with space-mode function $g_{a}^{2}=1 / 2$ (for space-mode function equal to unity), and the atomic transition frequency $\omega_{a}=100$ lead to $\Gamma_{a}=\pi$ and $\lambda_{a}=L / 50$. The first Poincaré recurrences appear at the time $t_{R}=2 \pi$. The upper cutoff on frequencies is set to $\omega_{\text {cut }}=200$; i.e., in the present simulation we use 400 modes of the electromagnetic field with the fundamental mode having the frequency $\omega_{1}=0.5$. In the figures presented in the paper we chose units such that the considered physical parameters are dimensionless.

during the first stage of the time evolution the decay rate does not depend on the position of the atom located around the center of the cavity.

For times large enough, the total excitation energy of the atom is transferred to the field, which in turn is effectively in a one photon (one excitation) state represented by two wave packets propagating towards the cavity mirrors. For finite cavities, at time approximately $L / 2 c$ the wave packets are reflected by the mirrors and at $t_{R} \simeq L / c$ they approach the atom which starts to re-absorb the energy from the field. We observe the reexcitation of the atom (i.e., a Poincare recurrence). In contrast to the "exponential" stage of the atomic decay, Poincaré recurrences are very sensitive to small position shifts of the atom within a wavelength of the resonant atomic transition. In Fig. 1 we clearly see that if the atom is positioned at the cavity center $\left(r_{1}=L / 2\right)$ then at time $t_{R}$ $\simeq L / c$ a very well pronounced Poincare recurrence of the atomic inversion takes place. One can say that at the moment when the Poincare recurrence appears the atom "sees" the cavity mirrors [27]. On the other hand, with a small shift of the atom from the cavity center to $\Delta r_{1}= \pm \lambda_{a} / 8$, the first atomic recurrence is almost completely suppressed. To understand this effect let us consider the two emitted wave packets (one to the left and one to the right) as monochromatic plane waves (at the atomic transition frequency and with the group velocity $c$ ). The difference of their geometrical paths is equal to $\lambda_{a} / 2$. This path difference results in destructive interference due to the accumulated phase difference of $\pi$. In other words, the atom does not "see" the wave packets reflected from the cavity mirrors. Obviously, when 
the two wave packets propagate further, then after the second reflection they accumulate a phase difference of $2 \pi$ so the corresponding Poincaré recurrence can then be seen (i.e., in this case the atom needs time twice as long to "see" the cavity mirrors).

When the atom is positioned at $\Delta r_{1}= \pm \lambda_{a} / 4$ the evolution of the atomic inversion is almost indistinguishable from the case where $\Delta r_{1}=0$. At the time of appearance of the first Poincare recurrence there is a constructive interference of the wave packets. The trivial phase shift $2 \pi$ results from the difference of the geometrical paths which is then equal to $\lambda_{a}$. With the atom at the position $\Delta r_{1}= \pm \lambda_{a} / 16$ the path difference of the two wave packets is equal to $\lambda_{a} / 4$ and the first Poincare recurrence is intermediate between the extreme cases $\left(\Delta r_{1}=0, \pm \lambda_{a} / 8\right)$ considered above. Dephasing of the wave packets by $\pi / 2$ results in a partially reduced reabsorption. We note that a more rigorous analysis should take into account the multimode structure of the wave packets as an additional source of dephasing, due to the different (Rabi) frequencies of the modes.

The second Poincare recurrence, associated with the second reflection of the wave packets from the cavity mirrors, starts in all cases shown in Fig. 1 at time $\simeq 2 t_{R}$. This is due to the fact that the optical paths of the wave packets are equal and they constructively interfere at the position of the atom.

We conclude that while the "exponential" character of the decay of the excited atom inside a large cavity is not influenced by small shifts of the atomic position, the first Poincaré recurrence is a position-dependent interference effect. This behavior can be explained using a simple argument: the phase-matching conditions necessary for the appearance of Poincaré recurrences can be associated with the phase factors $e^{-i t E_{k}}$ of the contributing eigenstates $\left|\Phi_{k}\right\rangle$ of the total Hamiltonian (7). Specifically, a Poincare recurrence can appear at time $t_{R}$ such that the relation $E_{k} t_{R} \simeq 2 \pi$ is valid for many values of $k$ (for more details, see [28]).

In Fig. 2 we present a stroboscopic set of plots describing the space-time evolution of the energy density of the cavity field. We assume the same situation as in Fig. 1. The atom is considered to be in the center of the cavity. We see two wave packets propagating to the right and to the left. Reflection of the wave packets from the cavity mirrors (at time $t \simeq L / 2 c$ ) is nicely demonstrated and the subsequent reexcitation of the atom is synchronized with the interference of the wave packets in the center of the cavity (compare with Fig. 1). These wave packets have "sharp" fronts propagating with the group velocity $c$. We note that the larger the number of modes coupled to the atom, the sharper the fronts are. The length of the tails of the wave packets depend on the lifetime of the atom.

\section{Spectrum of the cavity field}

Within the framework of cavity QED when the field interacting with the atoms is confined within ideal mirrors, there is nothing like a stationary regime which is necessary for the derivation of a time-independent spectrum of the field. The spectrum is intrinsically time dependent. In this case an operational definition of time-dependent spectrum can be given by the excitation probabilities of the cavity modes [see Eq. (14)].

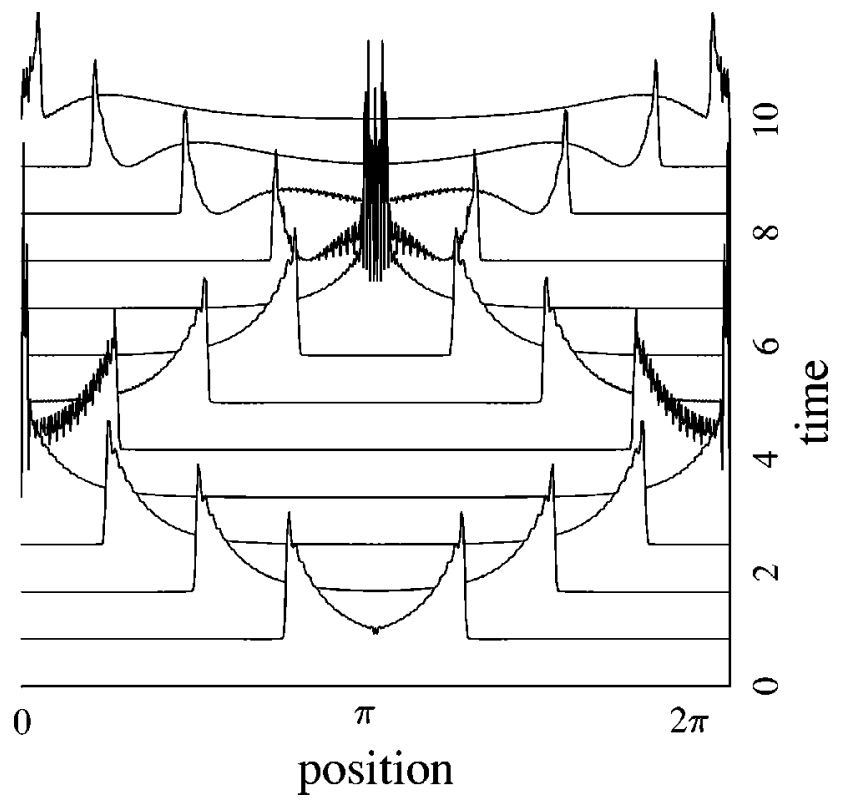

FIG. 2. A stroboscopic set of plots describing the space-time propagation of the mean energy density of the cavity field given by Eq. (15). We assume the same configuration and parameters as in Fig. 1 with the atom in the center of the cavity. We see that at the first stage of the time evolution the two wave packets are emitted and they propagate towards the cavity mirrors.

The spectrum of the cavity field is affected by the position of the atom. In particular, if the atom is located in the cavity center then the even modes are completely decoupled from the atom and only the odd modes can become excited [see Eq. (6)] establishing in this way oscillations in the spectrum of modes. However, at the point when the total excitation energy of the atom is transferred to the field, the envelope of the spectrum is "Lorentzian" irrespective of the position of the atom (providing that the decay is exponential).

On the other hand, it should be stressed that the spectrum of the interacting modes is highly transient even during the exponential decay period. It undergoes a gradual narrowing from a broad flat spectrum (initially all modes are in the vacuum state with the same probability) towards a Lorentzian-like line of width $\Gamma_{a}$. The narrowing is accompanied by transient oscillations of the spectral envelope. This transient behavior is illustrated in Fig. 3 which shows the spectral envelopes at different time moments during the exponential decay of the atomic excitation. At the time $t \approx 2$ the envelope of the cavity-field spectrum reaches its quasistationary shape, being very close to the corresponding (Lorentzian) emission spectrum usually associated with the freespace emission [1].

It is worth noticing that there is a close relation between the emission spectrum and the "spectrum" of squared scalar products (overlaps) between eigenvectors $\left|\Phi_{k}\right\rangle$ of the total Hamiltonian (7) and the given initial state $\left|\Psi\left(t_{0}\right)\right\rangle$, i.e.,

$$
S_{e}(k)=\left|\left\langle\Psi\left(t_{0}\right) \mid \Phi_{k}\right\rangle\right|^{2} \text {. }
$$

From Fig. 3 it is evident that the "spectrum" of overlaps (shown as - resembles the emission spectrum of the completely deexcited atom. In other words, the "spectrum" of overlaps offers an important time-independent characteriza- 

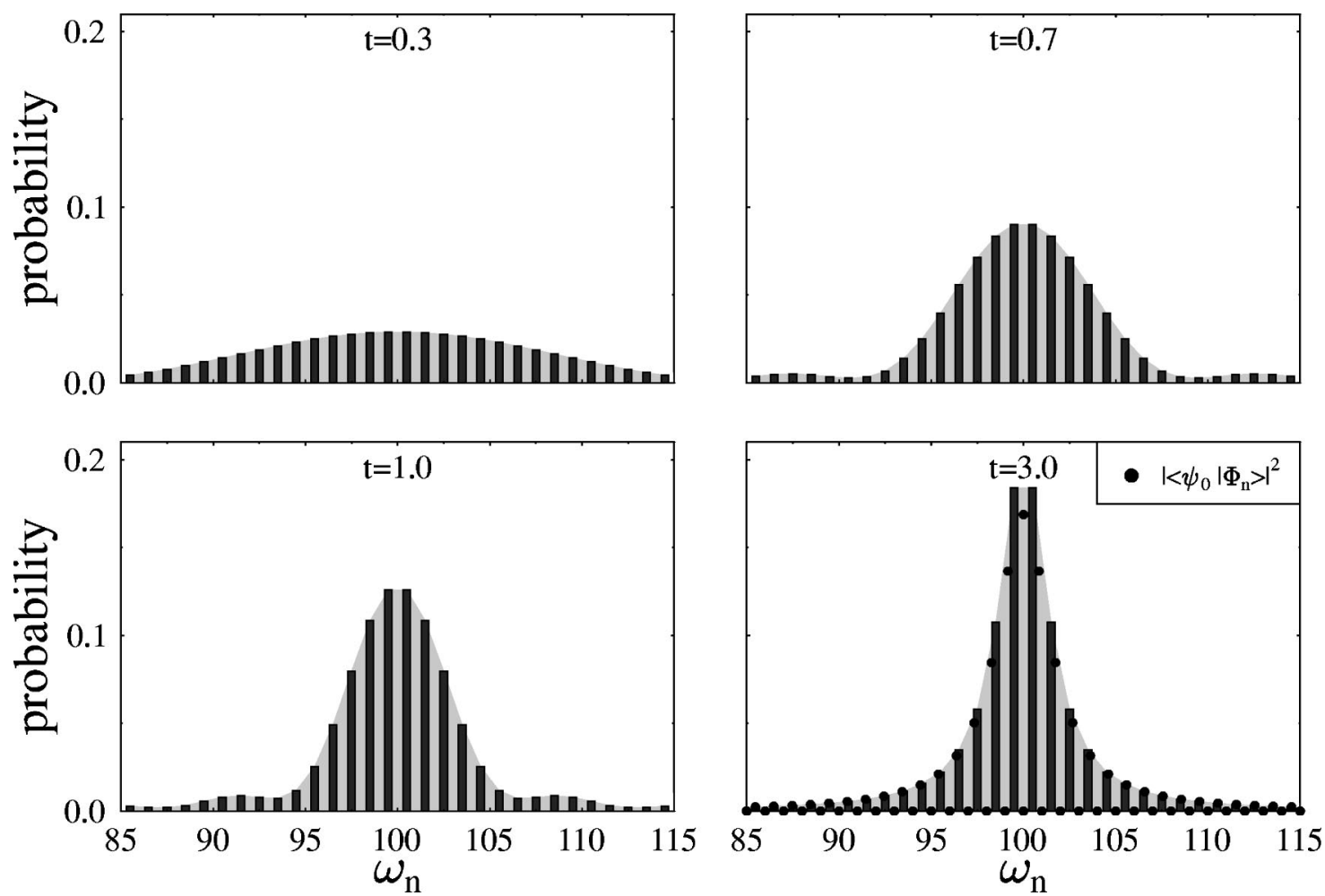

FIG. 3. Populations of cavity modes (i.e., the spectrum) at times $t=0.3,0.7,1,3$ for the atom located at the cavity center. The atom is initially prepared in its excited state and the cavity field is in the vacuum (for other conditions, see Fig. 1). For comparison purposes we show the overlaps of the eigenstates of the total Hamiltonian (7) with the initial state (these overlaps are denoted by the symbol 0 ). The ordering of eigenstates is given by their eigenvalues on the frequency axis. The even modes do not interact with the atom (see oscillations in the function $\left.\left|\left\langle\Psi_{0} \mid \Phi_{k}\right\rangle\right|^{2}\right)$.

tion of the system under consideration. If there exists a quasistationary spectrum of the cavity modes, it should mimic the "spectrum" of overlaps. In addition, a shift of the atomic transition frequency in the spectrum of eigenvalues can be associated with the energy shift.

Concluding this section, we note that our numerical calculations have been performed in the broadband approximation for the interaction constants given by Eq. (6), i.e., we have neglected the frequency dependence of the coupling constants replacing $\omega_{n}$ by $\omega_{a}$. This approximation is valid only for large enough cavities with $L \gg \lambda_{a}$ and "weak" interaction regimes with $\Gamma_{a} \ll \omega_{a}$.

A rather subtle point is the choice of the frequency cutoff. Strictly speaking, the model interaction Hamiltonian (5) with the interaction constants (6) within the broadband approximation leads in the second-order perturbation theory to $\log a$ rithmically divergent energy shifts for $\omega_{\text {cut }} \rightarrow \infty$ [24]. (Note that in our numerical calculations we have eliminated the shift of the excited atomic level by choosing a symmetrical frequency cutoff $\omega_{\text {cut }}=2 \omega_{a}$.) Without the broadband approximation, when the frequency dependence of the interaction constants (6) is taken into account, the energy shifts diverge linearly. It is well known [1,2] that if instead of the dipole approximation $\hat{H}_{\text {int }}=-\overrightarrow{\hat{d}} \cdot \overrightarrow{\hat{E}}$ we start with $\hat{H}_{\mathrm{int}}$ $=-\overrightarrow{\hat{p}} \cdot \overrightarrow{\hat{A}}$ then after the RWA is applied one obtains the interaction Hamiltonian (5) but with a different frequency dependence of the interaction constant, i.e.,

$$
g_{n}^{(j)}=\sqrt{\frac{\omega_{a}}{\omega_{n}}}\left(\frac{\omega_{a}}{\hbar \epsilon_{0} L}\right)^{1 / 2} d_{e g}^{(j)} \sin \left(k_{n} r_{j}\right) .
$$

In the broadband approximation the interaction constants given by Eq. (6) and Eq. (18) are identical and the results do not depend on the choice of the interaction Hamiltonian. On the other hand, without the broadband approximation the results are biased by the choice of the frequency dependence of the atom-field coupling. From the mathematical point of view, the coupling given by the expression (18) does not lead in second-order perturbation theory to divergent energy shifts for $\omega_{\text {cut }} \rightarrow \infty$. Obviously at the point when the two effective Hamiltonians considered above lead to different results, one has to be careful whether the model is physically relevant (for more details, see Ref. [2]).

\section{INHIBITION OF SPONTANEOUS EMISSION}

In the previous section we have considered the situation when the atom is "far" from the cavity mirrors [i.e., $\left.\min \left(r_{1}, L-r_{1}\right) \gg c / \Gamma_{a}\right]$ and the wave packets reflected by the mirrors do not directly affect the initial spontaneous decay of the atom. On the other hand, for distances between the atom and one of the cavity mirrors smaller than $c / \Gamma_{a}$ (here $1 / \Gamma_{a}$ is the spontaneous decay time in a free space) deviations from exponential decay should be expected [4-6]. In particular, the decay of a two-level atom which is positioned very close to the cavity mirror can be significantly suppressed. This effect is called the inhibition of spontaneous emission [4]. The inhibition of spontaneous emission is a positiondependent effect which is related to the position dependence of the atom-field coupling constant (6). In Fig. 4 we present numerical simulations for the time evolution of the popula- 


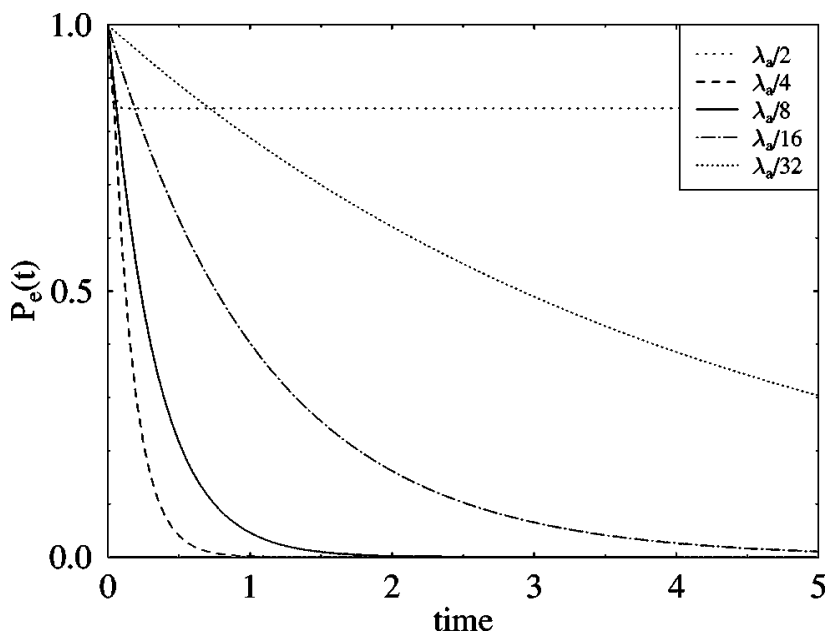

FIG. 4. The time evolution of the population $P_{e}(t)$ of the excited atomic level for the atom very close to the cavity mirror. The atom is considered at the following positions: $r_{1}=\lambda_{a} / 2$ (dotted line), $r_{1}=\lambda_{a} / 4$ (dashed line), $r_{1}=\lambda_{a} / 8$ (solid line), $r_{1}=\lambda_{a} / 16$ (dashed-dotted line), and $r_{1}=\lambda_{a} / 32$ (dotted line). The "reference", exponential decay of the atom at the cavity center $r_{1}=L / 2$ coincides with the case $r_{1}=\lambda_{a} / 8$. The initial conditions and other parameters are as in Fig. 1.

tion of the upper level of the atom described by the model interaction Hamiltonian (5). The atom is assumed to be initially in its excited state and the field in the vacuum. We consider several typical physical configurations. First, for reference, we plot the atomic decay of the atom positioned at $r_{1}=\lambda_{a} / 8$ (solid line) which is indistinguishable from the exponential decay of the atom at the cavity center (i.e., $P_{e}$ $\approx e^{-\Gamma_{a} t}$ for $\left.t \leqslant t_{R}\right)$. For other atomic positions $r_{1}=\lambda_{a} / 16$ and $r_{1}=\lambda_{a} / 32$ (here $\lambda_{a}=L / 50$ ) we clearly see that, the closer the atom is to the mirror, the slower the spontaneous decay is. The inhibition of spontaneous radiation is transparent for the considered positions of the atom. On the other hand, for a very specific atomic position close to the cavity mirror, the opposite effect-an enhancement of spontaneous emissiontakes place. Namely, for the distance $r_{1}=\lambda_{a} / 4$ of the atom from the cavity mirror, the atom decays as $P_{e} \approx e^{-2 \Gamma_{a} t}$, i.e., it radiates twice as fast compared with the free-space case.

In the context of our model the origin of the inhibition as well as the enhancement of spontaneous emission lies in the position dependence of the atom-field coupling (6). In particular, for $r_{1}=\lambda_{a} / 4$ the spatial-mode function $\sin \left(k_{n} r_{1}\right) \approx 1$ for all modes close to the resonant frequency $\omega_{a}$ irrespective of whether $n$ is even or odd. This means that the density of modes is increased by a factor of two compared with the case of the atom at the cavity center $r_{1}=L / 2$ [in this case, the modes with even $n$ are decoupled from the atom, i.e., $\sin \left(k_{n} L / 2\right)=0$ for even $n$ and $\sin \left(k_{n} L / 2\right)=1$ for odd $\left.n\right]$. The increased density of modes implies an enhancement of the spontaneous emission. In a similar way, when $r_{1}=\lambda_{a} / 8$ the spatial-mode function is $\sin \left(k_{n} r_{1}\right) \approx 1 / \sqrt{2}$ for all $n$ around the atomic transition frequency. This means that the coupling between the atom and the field mode is weaker. On the other hand, the density of the field modes is larger. The net effect in this case is that the spontaneous emission rate has the

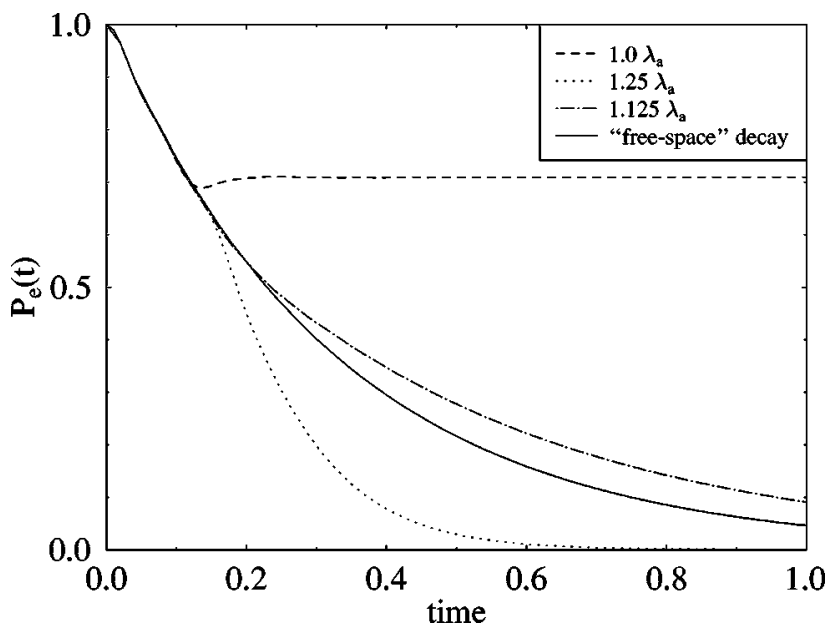

FIG. 5. The time evolution of the population $P_{e}(t)$ of the excited atomic level for the atom at the following positions: $r_{1}=\lambda_{a}$ (dashed line); $r_{1}=\lambda_{a}+\lambda_{a} / 4$ (dotted line); and $r_{1}=\lambda_{a}+\lambda_{a} / 8$ (dashed-dotted line) which are compared with the exponential decay of the atom (solid line). Other settings are the same as in Fig. 1. The suppression and the stimulation of the emission caused by the reflected wave packet are clearly seen.

value $\Gamma_{a}$ equal to the case when the atom is in the center of the cavity.

When the atomic distance from the cavity mirror is equal to one half of the atomic transition wavelength, i.e., $r_{1}$ $=\lambda_{a} / 2$, then all cavity modes around $\omega_{a}$ are essentially decoupled from the atom [now $\sin \left(k_{n} r_{1}\right) \approx 0$ ] which results in a dramatic inhibition of the spontaneous emission (see Fig. 4). In Fig. $4\left(r_{1}=\lambda_{a} / 16, \lambda_{a} / 32\right)$ the suppression of the spontaneous emission is clearly seen. The decay rate in these cases can be expressed as $\Gamma\left(r_{1}\right) \approx \Gamma_{a}\left[1-\cos \left(2 k_{a} r_{1}\right)\right]$. We note that in the case $r_{1}=\lambda_{a} / 16$ the atom decays completely while for $r_{1}=\lambda_{a} / 32$ the exponential decay law is interrupted by the Poincaré recurrence at $2 t_{R}$.

For distances of the atom from the cavity mirror larger than the wavelength of the resonant transition wavelength $\lambda_{a}$ the interference with the reflected wave packet can either stimulate or suppress the emission of the atom. To be specific we show in Fig. 5 the time evolution of the population of the upper level of the atom, which is positioned at three distances $r_{1}=\lambda_{a}$ (dashed line); $r_{1}=\lambda_{a}+\lambda_{a} / 4$ (dotted line); and $r_{1}=\lambda_{a}+\lambda_{a} / 8$ (dash-dotted line). For comparison purposes we plot also the usual exponential decay line. The phase accumulated by the wave packet during the round trip from the atom to the neighboring mirror and back is in the case $r_{1}=\lambda_{a}$ equal approximately to $5 \pi$ (here the additional contribution of $\pi$ is due to the reflection from the mirror), i.e., there is a destructive interference between the wave packet and the atom which results in the suppression of the radiation. On the other hand, when $r_{1}=\lambda_{a}+\lambda_{a} / 4$ the accumulated phase is approximately $6 \pi$, which leads to constructive interference. In this case the reflected wave packet, when it arrives at the position of the atom, starts to stimulate the atomic emission. In the units used in this simulation, the arrival time of the reflected wave packet is approximately $t$ $\simeq 0.16$ which coincides with the deviation from the initial exponential decay of the atom as seen in Fig. 5. When the 


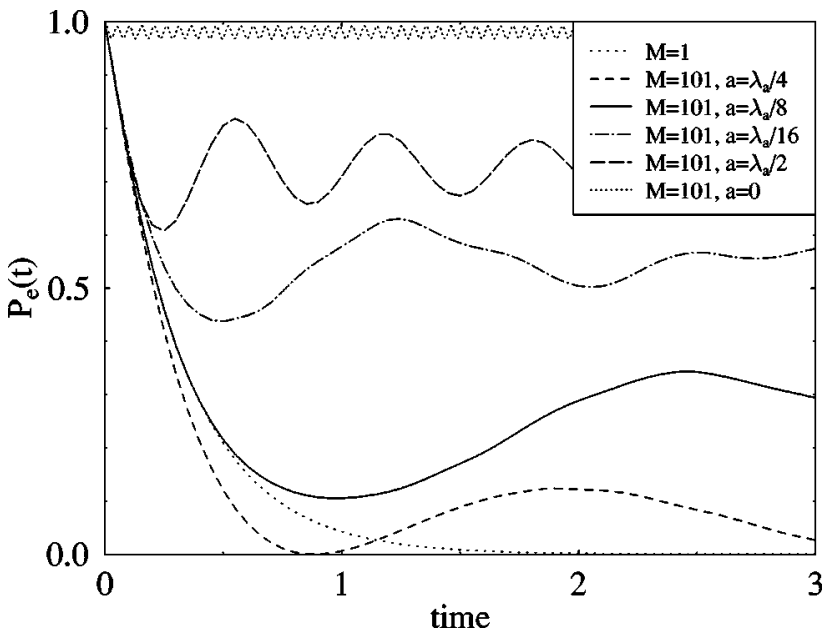

FIG. 6. The time evolution of the atomic excitation. We see the modification of the spontaneous emission of the atom at the cavity center surrounded by material media modeled as a set of two-level atoms (linear crystal lattice) initially prepared in the ground state. The cavity field is initially in the vacuum state. The regular linear crystal which fills the central part of the cavity is composed of $M$ $=101$ atoms with the interatomic distances $a=\lambda_{a} / 2$ (long dashed line), $a=\lambda_{a} / 4$ (short dashed line), $a=\lambda_{a} / 8$ (solid line), and $a$ $=\lambda_{a} / 16$ (dot-dashed line). In the case $a=0$ (dotted line) all atoms are positioned in the center of the cavity. The single atom exponential decay $M=1$ (sparse dotted line) is shown for reference.

position of the atom is $r_{1}=\lambda_{a}+\lambda_{a} / 8$, the accumulated phase of the reflected wave packet is $11 \pi / 2$ which gives rise to a partial suppression of radiation.

\section{SPONTANEOUS EMISSION IN MATERIAL MEDIA}

Atomic radiation can be significantly modified by the presence of other atoms in the cavity. Obviously, if the distance between the atoms is large enough then the exponential decay of the originally excited atom is not affected much. On the other hand, when the atoms are placed close together the situation is different (one of the consequences is a collective behavior of the atoms which might result in super-radiance; see, for instance, [29]).

In this section we consider a specific initial condition when the initially excited atom is surrounded by a collection of two-level atoms in the ground state. These additional atoms are considered as a material medium (e.g., a linear "crystal" composed of two-level atoms). By changing the density of the atoms we can also model systems such as atomic structures embedded in optical lattices (for interatomic distances comparable with the wavelength of the atomic transition) or dielectrics.

The modification of the spontaneous emission of the atom embedded in the material media modeled as a set of twolevel atoms is shown in Fig. 6. The regular crystal lattice built of $M=101$ atoms fills the central part of the cavity. Initially the excited atom is in the center of the cavity. The modification of the spontaneous emission depends on the interatomic distance $a$. When the "lattice" constant is $a$ $=\lambda_{a} / 2$ (long dashed line) we observe strong suppression of the spontaneous emission while for $a=\lambda_{a} / 4$ (short dashed

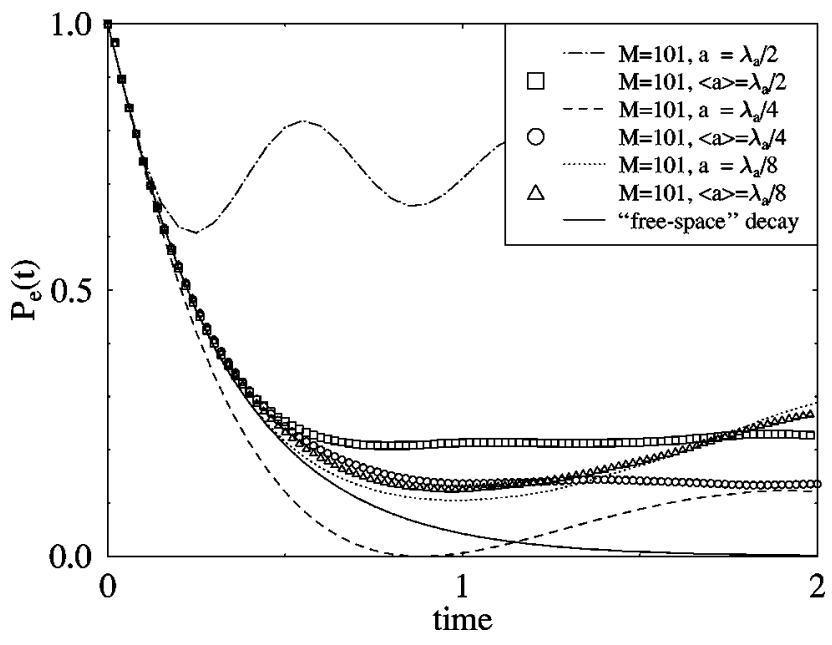

FIG. 7. The time evolution of the population $P_{e}(t)$ of the excited atomic level for the atom surrounded by identical atoms which form a random linear structure. Averages over 100 random configurations with one atom within the lattice constant $\langle a\rangle=\lambda_{a} / 8$ (triangles), $\langle a\rangle=\lambda_{a} / 4$ (circles), and $\langle a\rangle=\lambda_{a} / 2$ (squares) are compared with the corresponding regular lattices for $a=\lambda_{a} / 8$ (dotted line), $a=\lambda_{a} / 4$ (dashed line), and $a=\lambda_{a} / 2$ (dot-dashed line). Settings are as in Fig. 6 . The single-atom $(M=1)$ exponential decay (solid line) is shown for reference.

line) an enhancement of radiation compared with the single atom system (dotted line) takes place. The origin of this behavior is related to either destructive or constructive interference effects, respectively. From other examples, for $a$ $=\lambda_{a} / 8$ (solid line) and $a=\lambda_{a} / 16$ (dot-dashed line) it is seen that by increasing the density of the atoms of the medium the initially excited atom radiates more slowly. Moreover, the de-excitation is incomplete. That is, an increasing part of the excitation is captured by the initially excited atom. This subradiant behavior has already been analyzed for the extreme case when all atoms are located at the same position (e.g., the cavity center) [30]. The initial excitation is captured in the asymmetric atomic state and only a small part $\sim 1 / M$ is radiated into the cavity field.

The regular crystal lattice represents an idealized case. In fact, positions of the atoms can fluctuate due to various reasons (for example in the case of optical lattices with shallow wells formed from optical potentials). To simulate the situation when the atoms are not regularly distributed in the cavity we consider random configurations of the atoms. Specifically, the atoms are placed randomly such that within each lattice constant there is just one atom. Depending on the particular positions of the atoms, the dynamics of the originally excited atom can change dramatically. The atomic radiation can be either enhanced or suppressed. To obtain some effective "macroscopic" picture from our simulations, we have averaged our results over many random configurations-see Fig. 7. The dashed (dotted) line in this figure shows the time evolution of the atomic population of the initially excited atom when the atoms are regularly positioned with the lattice constant $a=\lambda_{a} / 4 \quad\left(a=\lambda_{a} / 8\right)$, representing enhancement (suppression) of radiation with respect to the "free-space" decay (solid line). The results of the numerical simulations corresponding to averaging over many 


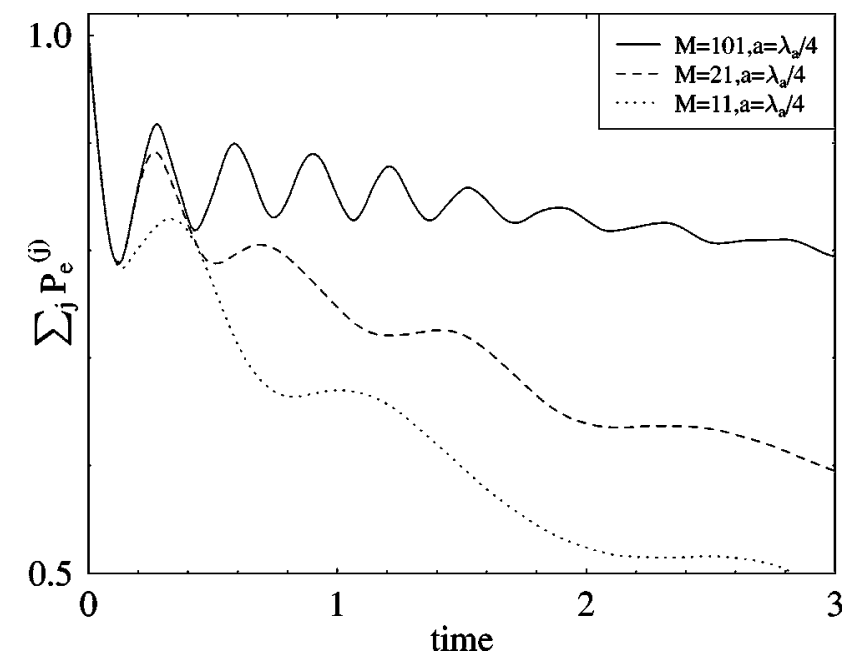

FIG. 8. The total excitation of the atoms $R_{\text {atoms }}=\Sigma P_{e}^{(j)}$ which form a regular linear lattice (same as in Fig. 6). We also consider two other regular configurations with the interatomic distance $a$ $=\lambda_{a} / 4$ and the number of atoms $M=11$ (dotted line) and $M=21$ (dashed line), respectively.

(100) random configurations of atoms are presented for the average distance between atoms $\langle a\rangle=\lambda_{a} / 4(\bigcirc)$ and $\langle a\rangle$ $=\lambda_{a} / 8(\triangle)$. In both cases the radiation of the atom is suppressed compared with the "free-space" decay. Another common feature of the dynamics in these cases is that the atom does not radiate away completely its initial excitation energy.

With the increase of the density of the atoms in the linear crystal lattice (e.g., for $a=\lambda_{a} / 16$ ) the differences between the regular lattice and the corresponding random lattice cases rapidly disappear.

For completeness we included in Fig. 7 also the case of the regular lattice with $a=\lambda_{a} / 2$ ( $\square$ ) and the average over random configurations with $\langle a\rangle=\lambda_{a} / 2$. Here the destructive interference which leads to the strong inhibition of the radiation in the case of the regular crystal, is deteriorated for random atomic configurations.

It is important to note that the modification of the spontaneous emission is a local effect, i.e., the atomic decay is influenced only by neighboring atoms. To check this we have performed simulations with only 11 atoms (i.e., one atom is excited and is surrounded by 10 atoms in the ground state). We have found that the radiation of the atom is essentially the same as in the case when the case $M=101$ is considered. Differences between the two cases occur only on a long time scale. This close-neighbor behavior is also seen from Fig. 8 where we plot the sum of atomic excitations $R_{\text {atoms }}=\sum P_{e}^{(j)}(t)$. We see that during the first decaying stage of the time evolution the excitation of the atoms is essentially the same for $M=11, M=21$ as well as for $M=101$. The oscillation patterns which we see reflect complex interference effects. Nevertheless one can trace a very general tendency in the picture - the linear lattice composed of twolevel atoms which surround the initially excited atom play the role of semitransparent mirrors placed very close to the atom. Therefore the results partially resemble the case of the single atom in the vicinity of a mirror (compare Fig. 6 with
Fig. 4). We study the microscopic model of mirrors and beam splitters composed of two-level atoms elsewhere [31].

\section{MASTER EQUATION FOR THE ATOM IN MATERIAL MEDIA}

The system of atoms and the field modes under consideration in an ideal cavity represents a closed system with unitary dynamics governed by the Schrödinger equation. In this section we will consider the decaying atom as an open system in the environment represented by field modes and other initially unexcited atoms. This analysis will provide us with a deeper insight into the problem of the emission of the atom in dielectrics. In general, an open system $S$ (in our case the atom which is initially excited) interacts with an environment $E$ (the other atoms surrounding the originally excited atom and the cavity modes) [32]. Let $\mathcal{H}_{S}$ denote a Hilbert space of the system $S$, and $\mathcal{H}_{E}$ is the Hilbert space associated with the environment $E$. The Hamiltonian $\hat{H}_{S E}=\hat{H}_{S} \otimes \hat{1}_{E}$ $+\hat{H}_{\text {int }}+\hat{1}_{S} \otimes \hat{H}_{E}$ of the composite system $S-E$ acts on $\mathcal{H}_{S} \otimes \mathcal{H}_{E}$. It is assumed that $S-E$ is a closed finitedimensional system which evolves unitarily. The density operator $\hat{\rho}_{S E}(t)$ of this composite system is governed by the von Neumann equation with the formal solution $\hat{\rho}_{S E}(t)$ $=\exp \left[-i\left(t-t_{0}\right) \hat{H}_{S E}\right] \hat{\rho}_{S E}\left(t_{0}\right) \exp \left[i\left(t-t_{0}\right) \hat{H}_{S E}\right]$, where the initial state is $\hat{\rho}_{S E}\left(t_{0}\right)=\hat{\rho}_{S}\left(t_{0}\right) \otimes \hat{\rho}_{E}\left(t_{0}\right)$ and $\hbar=1$. The reduced dynamics of the system $S$ is then defined as

$$
\hat{\rho}_{S}(t):=\hat{\mathcal{T}}\left(t, t_{0}\right) \hat{\rho}_{S}\left(t_{0}\right)=\operatorname{Tr}_{E}\left[\hat{\rho}_{S E}(t)\right]
$$

By definition, $\hat{\mathcal{T}}\left(t, t_{0}\right)$ is a linear map which transforms the input state $\hat{\rho}_{S}\left(t_{0}\right)$ onto the output state $\hat{\rho}_{S}(t)$. In our recent paper [33] we have addressed the question how to determine (reconstruct) the master equation which governs the time evolution of the reduced density operator $\hat{\rho}_{S}(t)$. It has been shown that this master equation can be written in the convolutionless form (we omit the subscript $S$ )

$$
\frac{d}{d t} \hat{\rho}(t)=\hat{\mathcal{L}}\left(t, t_{0}\right) \hat{\rho}(t)
$$

This is possible due to the fact that in the finite-dimensional Hilbert space, matrix elements of density operators are analytic functions. Consequently, $\hat{\mathcal{T}}\left(t, t_{0}\right)$ are nonsingular operators (except perhaps for a set of isolated values of $t$ ) in which case the inverse operators $\hat{\mathcal{T}}\left(t, t_{0}\right)^{-1}$ exist and the Liouvillian superoperator can be expressed as

$$
\hat{\mathcal{L}}\left(t, t_{0}\right):=\left[\frac{d}{d t} \hat{\mathcal{T}}\left(t, t_{0}\right)\right]-1\left(t, t_{0}\right) .
$$

We note that $\mathcal{T}\left(t, t_{0}\right)$ is uniquely specified by $\hat{H}_{S E}$ and by the initial state $\hat{\rho}_{E}\left(t_{0}\right)$ of the environment.

In Ref. [33] a general algorithm how to reconstruct the Liouvillian superoperator $\hat{\mathcal{L}}\left(t, t_{0}\right)$ from the knowledge of the unitary evolution of the composite $S-E$ system has been 
described. Once $\hat{\mathcal{L}}\left(t, t_{0}\right)$ is known then the master equation (20) is uniquely determined. The dynamics of the open system (in our case the atom) is given exclusively in terms of the system operators. Environmental degrees of freedom are completely eliminated from the reduced dynamics. Nevertheless, the state of the environment may change during the time evolution due to the interaction with the system. That is, there is no need to employ the assumption that the environment is a "big" reservoir which does not change under the action of the system.

It turns out that we can express the master equation for the originally excited atom as [33]

$$
\begin{aligned}
\frac{\partial}{\partial t} \hat{\rho}= & i \frac{\delta(t)}{2}\left[\hat{\rho}, \hat{\sigma}_{+} \hat{\sigma}_{-}\right] \\
& +\frac{\Gamma(t)}{2}\left[2 \hat{\sigma}_{-} \hat{\rho} \hat{\sigma}_{+}-\hat{\sigma}_{+} \hat{\sigma}_{-} \hat{\rho}-\hat{\rho} \hat{\sigma}_{+} \hat{\sigma}_{-}\right],
\end{aligned}
$$

where the time-dependent decay rate $\Gamma(t)$ and the timedependent dynamical energy shift $\delta(t)$ can be expressed through the probability amplitude $c_{1}(t)$ defined in Eq. (10) as

$$
\Gamma(t)=\operatorname{Re}[\eta(t)] ; \quad \delta(t)=\operatorname{Im}[\eta(t)]
$$

where

$$
\eta(t)=-2\left[\frac{1}{c_{1}(t)} \frac{d c_{1}(t)}{d t}\right]
$$

In general the parameter $\eta$ cannot be derived in an analytical form. In Fig. 9 we present results of numerical evaluation. We assume the initially excited atom to be in the center of the cavity. In the chosen units we obtain from the Fermi golden rule [see Eq. (16)] the decay rate $\Gamma_{a}=\pi$ (dashed line). We consider two cases: firstly, the case with just a single excited atom in the cavity (dotted line). Secondly, we assume that the excited atom is surrounded by 100 atoms (solid line) with regular spacing between atoms $\left(a=\lambda_{a} / 8\right)$. In the case of the single atom $\Gamma(t)$ oscillates around the value $\Gamma_{a}$. The amplitudes of these oscillations are relatively small. In fact, these oscillations become even smaller when we increase the density of modes. For large enough density of modes the decay rate $\Gamma(t)$ is approximately constant during the process of emission of the radiation. Its value is equal to the decay rate obtained from the Fermi golden rule [33]. This is true until the recurrence when the decay rate takes negative values (i.e., the atom starts to absorb energy from the field-compare with Fig. 1).

In the second case which corresponds to the decay of the atom in material medium the time evolution of $\Gamma(t)$ is more complex. At the initial stage of the time evolution $\Gamma(t)$ oscillates around the value $\Gamma_{a}$, but then it rapidly decreases. This corresponds to the suppression of radiation. From the figure we also see that at some stage $\Gamma(t)$ takes negative values-this is correlated with the absorption of energy from the wave packets reflected by surrounding atoms (see Fig. 6).

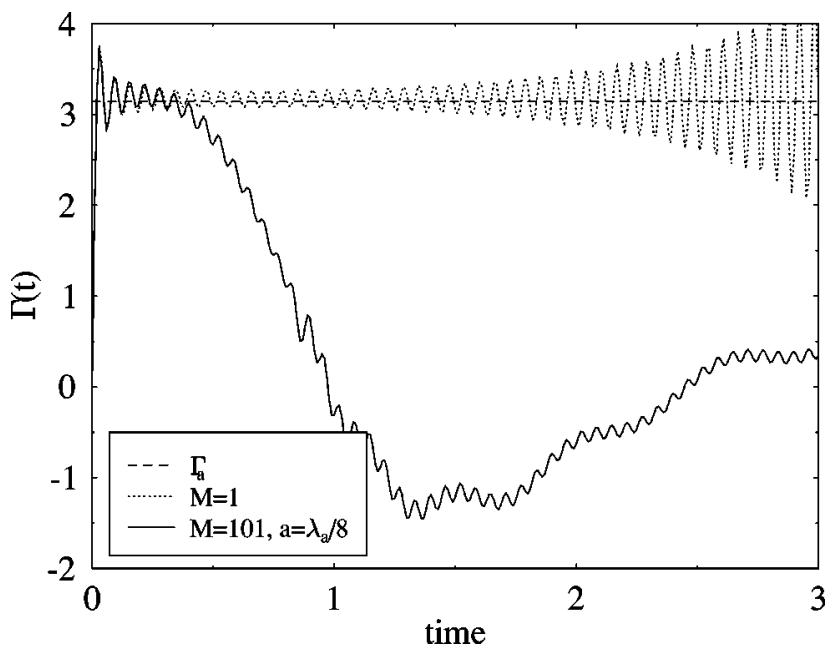

FIG. 9. The time evolution of the time-dependent decay rate $\Gamma(t)$. We assume the initially excited atom to be in the center of the cavity; other parameters are as in Fig. 1. In the chosen units we obtain from the Fermi golden rule the decay rate $\Gamma_{a}=\pi$ (see dashed line). We consider two cases: first, when there is just a single excited atom in the cavity (dotted line) and, second, when the excited atom is surrounded by 100 atoms (solid line) which create a linear crystal lattice with the regular spacing between atoms $\left(a=\lambda_{a} / 8\right)$.

We see that the effect of suppression of radiation in material media cannot be described with a simple substitution of the decay rate $\Gamma_{a}$ with a smaller constant.

\section{CONCLUSIONS}

In this paper we have presented a microscopic model describing dynamics of a two-level atom interacting with a multimode cavity field initially prepared in the vacuum state. We have analyzed the decay and re-excitation of the atom. In particular, we have shown that while the "exponential" character of the decay of the excited atom inside a large cavity is not influenced by small shifts of the atomic position, the first Poincaré recurrence is a position-dependent interference effect. We have also studied the time-dependent spectrum of the radiation emitted by the atom. We have associated the spectrum with the probabilities that cavity modes are excited. We have shown that this spectrum depends on the position of the atom in the cavity. In the quasistationary regime (when the excitation energy is completely transferred from the atom to the field) the spectrum has a Lorentzian-like envelope.

We have shown that the dynamics of the atom is dramatically changed when the atom is embedded in a material media modeled as a set of two-level atoms. Using this microscopic model we have shown that the spontaneous emission of the atom is not even approximately exponential. The atoms of the medium play a role of an imperfect mirror which partially reflects the emitted radiation back to the initially excited atom. The exponential decay in this case is altered by oscillations of the atomic population. The dynamics of the initially excited atom is very sensitive to particular positions of the "medium" atoms inside the cavity. In order to obtain an effective (macroscopic) picture, we have performed simu- 
lations with randomly distributed atoms and have averaged over many different configurations. We have shown that, on average the material medium causes the inhibition of radiation of the initially excited atom. We have derived the master equation describing the dynamics of the originally excited atom.

In this model we have studied mainly the process of decay of a single excited two-level atom. Within the framework of the same model one can study propagation of photon wave packets in material media (again modeled as a set of twolevel atoms). The model can be generalized to two dimen- sions and then one can simulate various optical networks in which optical elements are built up from two-level atoms and light fields are represented by photon wave packets (for more details, see Ref. [31]).

\section{ACKNOWLEDGMENTS}

We thank Rodney Loudon, Stig Stenholm, and Ed Hinds for helpful discussions. This work was supported by the Slovak Academy of Sciences, the GACR (201/98/0369), the Royal Society, and the European Union Network on Microlasers and Cavity Quantum Electrodynamics.
[1] C. Cohen-Tannoudji, J. Dupont-Roc, and G. Grynberg, AtomPhoton Interactions (John Wiley \& Sons, New York, 1992).

[2] P. W. Milonni, The Quantum Vacuum (Academic Press, New York, 1991).

[3] K. H. Drexhage, Prog. Opt. 12, 165 (1975).

[4] D. Kleppner, Phys. Rev. Lett. 47, 233 (1981).

[5] E. Hinds, in Advances in Atomic and Molecular Physics, Vol. 20, edited by D. Bates and B. Bederson (Academic Press, New York, 1985), p. 347.

[6] S. Haroche and D. Kleppner, Phys. Today 42 (1), 24 (1989); S. Haroche, in Fundamental Systems in Quantum Optics, edited by J. Dalibard, J.-M. Raimond, and J. Zinn-Justin (NorthHolland, Amsterdam, 1992).

[7] P. Meystre, in Progress in Optics, Vol. 30, edited by E. Wolf (North-Holland, Amsterdam, 1992).

[8] D. Meschede, Phys. Rep. 211, 201 (1992).

[9] For the state of the art of the cavity QED and for references, see papers in Advances in Atomic, Molecular and Optical Physics, edited by P. R. Berman (Academic Press, New York, 1994), Suppl. 2.

[10] We note here that the electromagnetic mode itself is specified by the "global" structure of the cavity. This means that even though the energy from the atom (the local particle) to the field is transferred locally, the particular field mode becomes "instantly", excited in the whole cavity.

[11] V. Weisskopf and E. Wigner, Z. Phys. 63, 54 (1930); see also earlier work by L. Landau, ibid. 45, 340 (1927) in which the exponential decay of an atomic state due to the spontaneous emission was described for the first time.

[12] J. Hamilton, Proc. Phys. Soc. London 59, 917 (1947). For a more detailed account of this approach, see also the monograph by C. Cohen-Tannoudji, J. Dupont-Roc, and G. Grynberg, Atom-Photon Interactions: Basic Processes and Applications (Wiley, New York, 1992).

[13] R. Davidson and J. J. Kozak, J. Math. Phys. 11, 189 (1970); 11, 1420 (1970); 12, 903 (1971); 14, 414 (1973); 14, 423 (1973); J. J.-W. Yang, R. Davidson, and J. J. Kozak, ibid. 15, 491 (1974).

[14] S. Swain, J. Phys. A 5, 1587 (1972); 5, 1601 (1972).

[15] S. A. Schelkunoff, Electromagnetic Waves (Van Nostrand, Princeton, 1943).

[16] E. M. Purcell, Phys. Rev. 69, 681 (1946).

[17] G. Barton, Proc. R. Soc. London, Ser. A 320, 251 (1970); 410, 141 (1987); 410, 175 (1987).
[18] It is well known (see, for instance, Ref. [28]) that a necessary condition for a pure exponential decay is that the energy spectrum of the electromagnetic field has no lower bound. Obviously, this is not the case of electromagnetic field neither in the free space nor in a cavity. Consequently, exponential decay is an approximation (but a very useful approximation). Deviations from exponential decay have been extensively discussed by many authors; see Refs. [13,14] and P. L. Knight and P. W. Milonni, Phys. Lett. 56A, 275 (1976).

[19] V. Bužek and M. G. Kim, J. Korean Phys. Soc. 30, 413 (1997).

[20] E. Yablonovitch, Phys. Rev. Lett. 58, 2059 (1987); S. John, ibid. 58, 2486 (1987).

[21] J. Opt. Soc. Am. B 10 (2) (1993), special issue on photonic band structures; J. Mod. Opt. 41, 171 (1994), special issue on photonic band structures.

[22] S. John and Tran Quang, Phys. Rev. A 52, 4083 (1995); Phys. Rev. Lett. 76, 1320 (1996); G. Kurizki, Phys. Rev. A 42, 2915 (1990); A. G. Kofman, G. Kurizki, and B. Sherman, J. Mod. Opt. 41, 353 (1994); M. Konôpka, Acta Phys. Slov. 48, 77 (1998); J. Mod. Opt. 46, 15 (1999); M. Konôpka and V. Bužek, e-print quant-ph/9901069.

[23] S. Stenholm, Phys. Rep. 6, 1 (1973).

[24] P. W. Milonni, Phys. Rep. 25, 1 (1976).

[25] E. T. Jaynes and F. W. Cummings, Proc. IEEE 51, 89 (1963). For a recent review, see B. W. Shore and P. L. Knight, J. Mod. Opt. 40, 1195 (1993).

[26] W. H. Press, S. A. Teukolsky, W. T. Vetterling, and B. P. Flannery, Numerical Recipes in C, 2nd ed. (Cambridge University Press, Cambridge, England, 1995), Chap. 16.1.

[27] J. Parker and C. R. Stroud, Jr., Phys. Rev. A 35, 4226 (1987); H. Gießen, J. D. Berger, G. Mohs, P. Meystre, and S. F. Yelin, ibid. 53, 2816 (1996).

[28] P. W. Milonni, J. R. Ackerhalt, H. W. Galbraith, and Mei-Li Shih, Phys. Rev. A 28, 32 (1983), and references therein.

[29] R. H. Dicke, Phys. Rev. 93, 99 (1954).

[30] V. Bužek, Czech. J. Phys., Sect. B 39, 345 (1989); Phys. Rev. A 39, 2232 (1989); S. M. Barnett, B. Huttner, R. Loudon, and R. Matloob, J. Phys. B 29, 3763 (1996), and references therein.

[31] M. Havukainen, G. Drobný, S. Stenholm, and V. Bužek, Los Alamos e-print archive quant-ph/9902078.

[32] E. B. Davies, Quantum Theory of Open Systems (Academic Press, London, 1976).

[33] V. Bužek, Phys. Rev. A 58, 1723 (1998). 